SPICA Workshop, 04003 (2009)

DOI:10.1051/spica/200904003

(C) Owned by the authors, published by EDP Sciences, 2009

This is an Open Access article distributed under the terms of the Creative Commons Attribution-Noncommercial License, which permits unrestricted use, distribution, and reproduction in any noncommercial medium, provided the original work is properly cited.

\title{
NEXT GENERATION STUDY OF THE COSMIC METALLICITY EVOLUTION WITH SPICA
}

\author{
T. Nagao ${ }^{1}$, R. Maiolino ${ }^{2}$, and H. Matsuhara ${ }^{3}$ \\ ${ }^{1}$ Graduate School of Science and Engineering, Ehime University, 2-5 Bunkyo-cho, Matsuyama 790-8577, Japan \\ ${ }^{2}$ INAF - Osservatorio Astronomico di Roma, via di Frascati 33, 00040 Monte Porzio Catone, Italy \\ ${ }^{3}$ Institute of Space and Astronautical Science, Japan Aerospace Exploration Agency, Sagamihara, Kanagawa 229-8510, Japan
}

\section{Abstract}

In order to explore the metallicity of high- $z$ dusty galaxies in the evolving phase, mid- and far-infrared line diagnostics by using deep spectroscopy with SPICA are extremely powerful. We here propose to use far-infrared [O $\mathrm{III}]$ and [N III] lines to study the metallicity in dustenshrouded parts in galaxies.

Key words: Galaxies: evolution - Infrared: spectroscopy Missions: SPICA

\section{INTRODUCTION}

Measuring the metallicity of galaxies at various redshifts is a powerful way to give constraints on the galaxy evolutionary scenarios, since the chemical properties of galaxies are the result of their past cumlative star-formation. Especially the tight mass-metallicity relation of galaxies (i.e. more massive galaxies have higher metallicity; hereafter MZR) is important, because the MZR suggests more efficient loss of created metals thgough galactic superwind phenomena in less-massive galaxies (e.g., Tremonti et al., 2004). A more interesting issue is the redshift evolution of the MZR. Maiolino et al. (2008) reported a massdependent evolution of the MZR between $0<z<3$; i.e., less-massive galaxies tend to shows gradual metallicity evolution in this redshift range while massive galaxies completed their metallicity evolution at earlier cosmic epoch (see also, e.g., Savaglio et al., 2005; Erb et al., 2006) This may corresponds to the chemical version of the gdown-sizingh galaxy evolution.

However, all of the above studies are for "alreadyevolved" galaxies; i.e., dust-unreddened populations. Recently it is pointed out that Ultra-luminous Infrared Galaxies (ULIRGs) are significantly deviated from the standard MZR (Rupke et al., 2008; Caputi et al., 2008). Since the ULIRGs are in a rapidly evolving phase, MZRs (and its redshift evolution) for ULIRGs will give strong constraints on the galaxy evolutionary scenarios. Such studies are evidently important especially at high redshifts, where a large fraction of star formation occurred in ULIRGs (e.g., Le Floc'h et al., 2005). Note that the current metallicity measurements for ULIRGs are still relying on rest-frame opti- cal emission lines, and thus the dust-enshrouded parts in ULIRGs have not yet examined.

\section{Method: Far-infrared Metallicity Diagnostics}

A breakthrough in this issue will be achieved through far-infrared (FIR) metallicity diagnostics for high- $z$ dusty star-forming galaxies with using SPICA/SAFARI. Among various potential metallicity diagnostics, emission-line flux ratios using [N III] $57.2 \mu \mathrm{m}$ are promising. Specifically, the flux ratio of [O III ] $51.8 \mu \mathrm{m} /[\mathrm{N}$ III] $57.2 \mu \mathrm{m}$ is very sensitive to the gas metallicity. The simultaneous use of $[\mathrm{O}$ III] $88.3 \mu \mathrm{m}$ in addition to [O III] $51.8 \mu \mathrm{m}$ and [N III] $57.2 \mu \mathrm{m}$ is even better since the density dependence of the diagnostic ratio is well corrected. This method has an advantage with respect to other near and mid-infrared metallicity diagnostics using hydrogen lines (e.g., Verma et al., 2003), since the IR hydrogen lines such as Pfunt and Humphreys series lines are generally very weak. The method using [O III] and [N III] lines has been already applied to the ISO data to infer the metallicity of Galactic planetary nebulae (e.g., Liu et al., 2001). The superb sensitivety of SPICA/SAFARI enables us to extend such an approach toward the extragalactic universe. We already confirmed that the flux ratio of [O III] $51.8 \mu \mathrm{m}+88.3 \mu \mathrm{m}$ $/[\mathrm{N} \mathrm{III}] 57.2 \mu \mathrm{m}$ works very well as a metallicity diagnostic tool also for star-forming galaxies, based on detail photoionization model calculations (Nagao, Maiolino, et al., in prep.). Note that these three lines are the strongest emission from HII regions in galaxies (i.e., excluding PDR lines such as [O I] and [C II], because they comes from different regions).

\section{Detection Feasibility}

In Figure 1 fluxes of these fine structure lines at various redshifts (M82 spectrum is assumed) are shown together with the SPICA sensitivities. The [N III] $57.2 \mu \mathrm{m}$ line (faintest one among the three lines) from galaxies whose star-formation rate (SFR) is similar to M82 (Colbert et al., 1999) can be detected up to $z \sim 0.4$ with SAFARI ( $5 \sigma$ detection with one hour exposure). However, since ULIRGs (that are our main targets) have $\sim 2$ order higher SFRs than M82, we will detect [N III] $57.2 \mu \mathrm{m}$ (and two [O III] lines simultaneously) from ULIRGs at up to $z \sim 2$. This redshift range is limited by both flux sensitivity and the 
wavelength coverage of SAFARI. Note that a wavelength resolution of $\sim 1000$ is adequate for this science, taking a typical emission-line velocity width of star-forming galaxies to be $\sim 300 \mathrm{~km} \mathrm{~s}^{-1}$ into account. In addition, if BLISS is available, one can study even LIRGs (with the total infrared luminosity of order of $\left.10^{11} L_{\odot}\right)$ at up to $z \sim 2$.

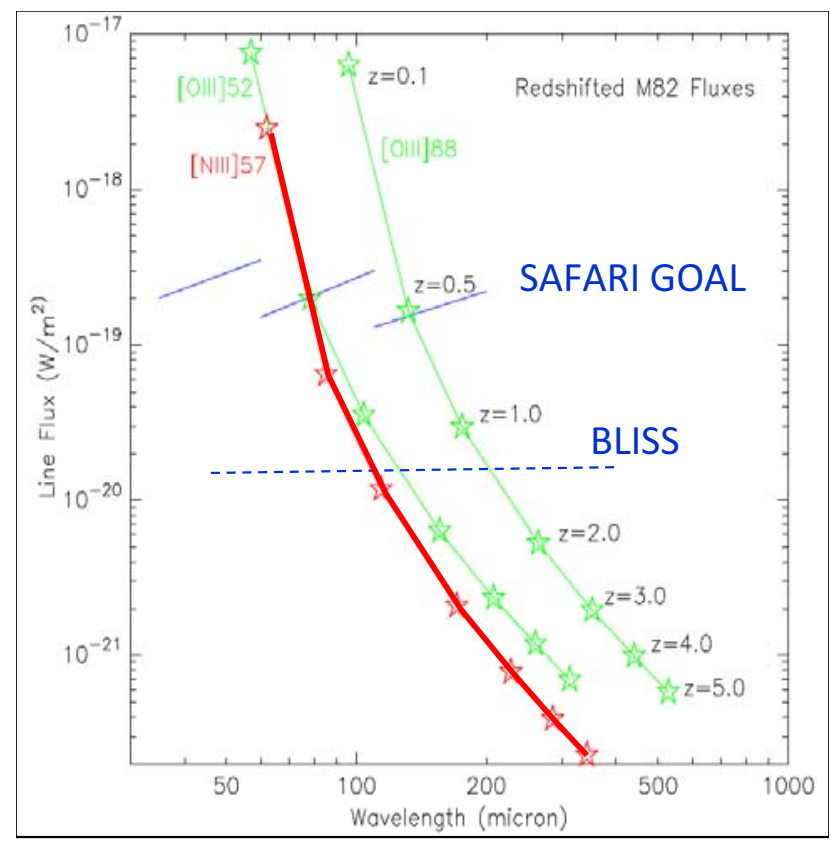

Figure 1. Line fluxes of redshifted [O III] and [N III] lines (thin and thick solid curves), assuming the M82 spectrum (Colbert et al., 1999). SPICA/SAFARI (GOAL) and BLISS sensitivity limits (1 hour, $5 \sigma$ ) are shown by solid and dashed lines, respectively. Note that our main targets are not M82-like galaxies but ULIRGs, whose SFR reaches $\sim 100$ times higher than that of M82.

Summarizingly, SPICA/SAFARI can measure the metallicity of ULIRGs based on reddening-insensitive FIR lines of the [O III] and [N III] transitions, up to $z \sim 2$. This is crucial to examine the redshift evolution of the MZR for rapidly-evolving galaxies, that will then contribute to give strong constraints on the galaxy evolutionary scenarios.

\section{ACKNOWLEDGEMENTS}

We thank colleagues of SPICA science working group members, especially Toru Yamada and Takashi Ichikawa, for their constructive comments and discussions.

\section{REFERENCES}

Caputi, K. I., et al. 2008, ApJ, 680, 939

Colbert, J. W., et al. 1999, ApJ, 511, 721

Erb, D. K., Shapley, A. E., Pettini, M., Steidel, C. C.,

Reddy, N. A., \& Adelberger, K. L. 2006, ApJ, 644, 813

Le Floc'h et al. 2005, ApJ, 632, 169

Liu, X.-W., et al. 2001, MNRAS, 323, 343

Maiolino, R., et al. 2008, A\&A, 488, 463
Rupke, D. S. N., Veilleux, S., \& Baker, A. J. 2008, ApJ, 674,172

Savaglio, S., et al. 2005, ApJ, 635, 260

Tremonti, C. A., et al. 2004, ApJ, 613, 898

Verma, A., Lutz, D., Sturm, E., Sternberg, A., Genzel, R., \& Vacca, W. 2003, A\&A, 403, 829 